\title{
Capacity of Band Limited Wavefields Observed Over Finite Spatial and Temporal Windows
}

\author{
Farhana Bashar, Thushara D. Abhayapala and S. M. Akramus Salehin \\ Research School of Engineering \\ The Australian National University \\ Canberra 0200 ACT, Australia
}

\begin{abstract}
In this work, we study the channel capacity for broadband transmission in a multipath wavefield observed on the surface of a limited source-free region over a finite temporal window. It has a wide range of applications, such as characterizing broadband distributed or large scale MIMO systems and evaluating the capacity of these systems. In order to address this topic, for given constraints on region size, bandwidth and observation time, the multipath field is characterized as a series of orthogonal basis functions encoded in a finite number of spatial modes. Each of these spatial modes represents an independent channel. We show that for each channel, how the observable time and band limited signals are coupled to a limited region of space. Afterwards, analogous to Shannon's work, we evaluate the capacity of each channel. The derived result indicates that due to the space-frequency coupling relation, for a fixed average signal power over the bandwidth, not all channels have the same capacity. Rather, the lower spatial modes provide large performance gains and channel capacity drops as spatial mode index increases. In addition, different modes provides optimum performance at different radial positions.
\end{abstract}

Index Terms-Shannon's capacity, distributed networks, large scale MIMO.

\section{INTRODUCTION}

Multi-antenna systems exploit spatial diversity (for instance, MIMO communications) to provide several independent channels over which information can be transmitted. Hence, the study of the channel capacity of multi-antenna systems is an area of ongoing investigation. There are many approaches to characterize the channel capacity of multi-antenna systems in literature [1]-[8]. Moreover, recently, distributed MIMO communication has emerged as a promising tool to achieve large performance gains in wireless networks by cooperating information between transmit and receive clusters. The work of [9] stated the capacity scaling of narrowband distributed MIMO systems in a line-of-sight propagation environment. However, practical wireless transmissions are performed in scattering environments and also the transmitted signals are not narrowband. Considering this, we aim to evaluate the information capacity available for users in a limited spherical region cooperating to receive broadband information. We envisage that the results presented in this work will be useful in characterizing broadband distributed or large scale MIMO systems in scattering environments and evaluating the capacity of these systems.

The analysis of this work builds upon extending the results of Shannon [10] to spatial domain in addition to the time- frequency domain. In order to do so, we study a band limited multipath wavefield observed on the surface of a limited source-free region of space over a finite time window. We assume that the multipath field is generated by transmitting antennas and scatterers that exist outside the observable region of space. The signals observable within this wavefield are characterized as a series of orthogonal basis functions encoded in a finite number of spatial modes. From the spatial diversity perspective, each of these modes represents an independent channel. Then analogous to Shannon's work [10], for each channel, we (i) evaluate the signal degrees of freedom ${ }^{1}$, (ii) represent the signals observed within the channel geometrically and (iii) calculate an upper bound to the information capacity. We derive the capacity for each spatial mode assuming that the average power of the transmitted signals at different spatial modes and frequencies is constant and equal. Our result shows how the capacity at a particular radius is distributed over the different spatial modes for broadband communications. The simulation results indicate that different spatial modes provide optimum capacity at different radial positions.

\section{System Model}

Consider a wavefield band limited to $\left[F_{0}-W, F_{0}+W\right]$ and observed on the surface of a spherical region of radius $R$ over a time window $[0, T]$. Here, $F_{0}$ represents the mid band frequency. Any signal sampled or recorded on this region of space is generated by transmitting antennas and scatterers that exist outside the region of interest.

We denote the observable space-frequency spectrum at the edge of the spherical region of radius $\|\boldsymbol{x}\|=R$ operating at a particular frequency $\omega$ as $\Psi(R \hat{\boldsymbol{x}}, \omega)$. Here, $\hat{\boldsymbol{x}} \triangleq \boldsymbol{x} /\|\boldsymbol{x}\|$ is the unit vector in the direction of nonzero vector $x$. The spacefrequency spectrum $\Psi(R \hat{\boldsymbol{x}}, \omega)$ can be expressed as a scalar wavefield as [13, p. 227]

$$
\Psi(R \hat{\boldsymbol{x}}, \omega)=\sum_{n=0}^{\infty} \sum_{m=-n}^{n} \Psi_{n m}(R, \omega) Y_{n m}(\hat{\boldsymbol{x}})
$$

where the spatial mode $n(\geq 0)$ and the spatial order $m(|m| \leq$ $n)$ are integers, such that for any particular mode $n$, there are $2 n+1$ orders, $\Psi_{n m}(R, \omega)$ is the $(n, m)^{t h}$ mode spacefrequency spectrum and $Y_{n m}(\cdot)$ are the spherical harmonics.

\footnotetext{
${ }^{1}$ Detailed studies for degrees of freedom of $2 D$ and $3 D$ wavefields were presented in [11], [12], respectively.
} 
Further, the $(n, m)^{t h}$ mode space-frequency spectrum can be expressed as the product of the $(n, m)^{t h}$ mode signal spectrum $\alpha_{n m}(\omega)$ and the spherical Bessel functions of the first kind $j_{n}(\omega R / c)$ as [13, p. 227]

$$
\Psi_{n m}(R, \omega) \triangleq \alpha_{n m}(\omega) j_{n}\left(\frac{\omega}{c} R\right) .
$$

In this work, we consider that the $(n, m)^{t h}$ mode signal spectrum $\alpha_{n m}(\omega)$ is limited to a certain average power $P$ irrespective of modes $n$, orders $m$ and frequencies $\omega$, i.e.,

$$
E\left\{\left|\alpha_{n m}(\omega)\right|^{2}\right\} \leq P, \quad \forall n, m, \omega
$$

where $E\{\cdot\}$ represents the expectation operation.

\section{Signal Degrees of FreEdom Result}

In this section, we evaluate the available degrees of freedom of the $(n, m)^{t h}$ mode signal observed within a wideband multipath field over a finite time interval on the surface of a spherical region. Before proceeding to do so, we evaluate the observation time of time limited signals at each spatial mode $n$.

\section{A. Observation time of the $(n, m)^{\text {th }}$ Mode}

Let $\psi_{n m}(R, t)$ and $a_{n m}(t)$ be the inverse Fourier transform of the $(n, m)^{t h}$ mode space-frequency spectrum $\Psi_{n m}(R, \omega)$ and the $(n, m)^{t h}$ mode signal spectrum $\alpha_{n m}(\omega)$, respectively. Consider the $(n, m)^{t h}$ modal wavefront propagating across the observation sphere, observing $\psi_{n m}(R, t)$ over a time window $[0, T]$ at radius $R$ is equivalent to observing $a_{n m}(t)$ over a time window $[-R / c, T+R / c]$. In effect, the $(n, m)^{t h}$ mode space-time signal $\psi_{n m}(R, t)$ captures information content of the $(n, m)^{t h}$ mode time domain signal $a_{n m}(t)$ over an effective time interval

$$
T_{\text {eff }}=T+\frac{2 R}{c}
$$

which is independent of the spatial mode index $n$.

A detailed discussion on the observation time of the $(n, m)^{t h}$ mode space-time signal is provided in [11, Theorem 1] [12, Theorem 1] for $2 D$ and $3 D$ wavefields, respectively.

\section{B. Bandwidth of the $(n, m)^{\text {th }}$ Mode}

We show that depending on the mode index $n$, the effective bandwidth of the space-frequency spectrum varies from the given bandwidth. We show this below with a rigorous analysis of the $(n, m)^{t h}$ mode space-frequency spectrum $\Psi_{n m}(R, \omega)$ (2).

We can observe from Fig. 1 that the $0^{\text {th }}$ order spherical Bessel function $j_{0}(z)$ is active within the range $z \in[0, \infty)$. On the contrary, for a given $z$, the spherical Bessel functions $j_{n}(z)$ start small (in the order of zero) as $n$ increases. For $n>0$, this observation is supported by the following asymptotic form [15, eqn. 13]

$$
j_{n}(z) \leq \frac{\sqrt{\pi}}{2}\left(\frac{z}{2}\right)^{n} \frac{1}{\Gamma(n+3 / 2)}, \quad z \ll n
$$

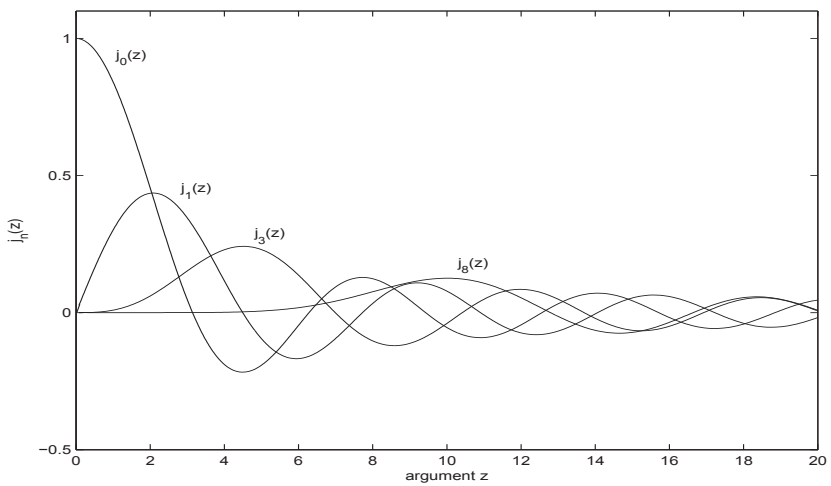

Fig. 1. Spherical Bessel functions of first kind $j_{n}(z)$ vs. argument $z$ for $n=0,1,3,8$.

where $\Gamma(\cdot)$ is the Gamma function.

According to this bound, in (2), for the $(n, m)^{t h}$ mode, when argument $\omega R / c \ll n$, magnitude of $j_{n}(\omega R / c)$ is significantly small. Thus, the magnitude of the space-frequency spectrum becomes trivial. Therefore, magnitude of $j_{n}(\omega R / c)$ has to be at least equivalent to some threshold $\gamma$. Lets assume that at a fixed radius $R, \omega_{n}$ is the maximum angular frequency below which the magnitude of the spherical Bessel function $j_{n}(\omega R / c)$ is less than the threshold $\gamma$. Therefore, substituting $z$ by $\omega_{n} R / c$ in (5), we obtain

$$
j_{n}\left(\frac{\omega_{n}}{c} R\right)=\gamma \approx \frac{\sqrt{\pi}}{2}\left(\frac{\omega_{n} R}{2 c}\right)^{n} \frac{1}{\Gamma(n+3 / 2)} .
$$

Now, using the Stirling lower bound for the Gamma functions $\Gamma(n+3 / 2)>\sqrt{2 \pi} e^{-(n+1 / 2)}(n+1 / 2)^{n+1}$, we rewrite (6) as $\beta e^{\left(e \omega_{n} R / 2 c-n\right)} \leq \gamma$ where $\beta=(e / \sqrt{8}) n^{n}(n+1 / 2)^{-(n+1)}$. This result is obtained using the exponential inequality, $(1+x / n)^{n} \leq$ $e^{x}$ for $n \neq 0$. Since $\beta<1$, for $n>0$ and $\omega_{n}=2 \pi F_{n}$, we have

$$
e^{\left(e \pi F_{n} R / c-n\right)}<\gamma
$$

We define $F_{n}$ as the critical frequency. Now, making $F_{n}$ the subject of the formula in (7), the maximum value of the critical frequency for the $n^{t h}$ mode is

$$
F_{n}=\frac{n c}{e \pi R}+\frac{c}{e \pi R} \log \gamma
$$

such that at radius $R$, above this critical frequency $F_{n}$ magnitude of the spherical Bessel function $j_{n}(\omega R / c)$ is greater than a predefined threshold $\gamma$.

Note that the $(n, m)^{t h}$ mode signal spectrum $\alpha_{n m}(\omega)$ (2) is band limited over the range $\left[F_{0}-W, F_{0}+W\right]$, since the multipath field is band limited to $\left[F_{0}-W, F_{0}+W\right]$. Moreover, as depicted in Fig. 1, the spherical Bessel functions $j_{n}(z)(n>$ $0)$ start more slowly as $n$ increases. Thus, beyond a certain spatial mode $n$, the critical frequency $F_{n}>F_{0}-W$ and the useable bandwidth of the modes above varies from the given frequency bandwidth. Further, if we keep increasing $n$, beyond a certain mode $n, F_{n}>F_{0}+W$ and the useable bandwidth of the modes above is zero. Hence, the effective frequency 
bandwidth of the $(n, m)^{t h}$ mode space-frequency spectrum $\Psi_{n m}(R, \omega)$ is

$$
W_{n}= \begin{cases}2 W, & 0 \leq n \leq N_{\min } \\ F_{0}+W-F_{n}, & N_{\min }<n \leq N_{\max } \\ 0, & \text { otherwise }\end{cases}
$$

Here, the critical frequency $F_{n}$ is defined in (8), $N_{\min }$ is the lowest spatial mode beyond which the critical frequency $F_{n}>F_{0}-W$ and $N_{\max }$ is the lowest spatial mode beyond which the critical frequency $F_{n}>F_{0}+W$.

\section{Degrees of Freedom of the $(n, m)^{\text {th }}$ Mode Signal}

We are now in a position to set up the mathematical formalism to evaluate the degrees of freedom of the $(n, m)^{t h}$ mode space-time signal observed over a frequency range for a finite temporal window at the edge of the spherical region. In order to do so, it is useful to think of $(n, m)^{t h}$ mode spacefrequency spectrum $\Psi_{n m}(R, \omega)$ at radius $R$ in time domain as

$$
\psi_{n m}(R, t)=\frac{1}{2 \pi} \int_{\Omega_{n}} \Psi_{n m}(R, \omega) e^{j \omega t} d \omega
$$

where the integration is taken over $\Omega_{n}$ with $\Omega_{n} \in\left[2 \pi\left(F_{0}-\right.\right.$ $\left.W), 2 \pi\left(F_{0}+W\right)\right]$ for $0 \leq n \leq N_{\min }$ and $\Omega_{n} \in\left[2 \pi F_{n}, 2 \pi\left(F_{0}+\right.\right.$ $W)$ ] for $N_{\min }<n \leq N_{\max }$ where $F_{n}$ is defined in (8).

Lets replace $t$ in (10) by $t=\frac{\ell}{W_{n}}$ where $\ell$ is any positive or negative integer. Hence, we obtain

$$
\psi_{n m}\left(R, \frac{\ell}{W_{n}}\right)=\frac{1}{2 \pi} \int_{\Omega_{n}} \Psi_{n m}(R, \omega) e^{j \omega \frac{\ell}{W_{n}}} d \omega .
$$

Note that on the left side, we have the values of the $(n, m)^{t h}$ mode space-time signal $\psi_{n m}(R, t)$ at sampling points in time domain, whereas, on right side, we have the $\ell^{\text {th }}$ coefficients in the Fourier series expansion of the $(n, m)^{t h}$ mode spacefrequency spectrum $\Psi_{n m}(R, \omega)$. According to the uniqueness property of Fourier transform, $\psi_{n m}(R, t)$ is determined completely by its samples, and analogous to Shannon's work [10], we can reconstruct $\psi_{n m}(R, t)$ from its samples as

$$
\psi_{n m}(R, t)=\sum_{\ell=-\infty}^{\infty} \psi_{n m}\left(R, \frac{\ell}{W_{n}}\right) \phi_{n \ell}(t)
$$

where

$$
\phi_{n \ell}(t)=e^{j 2 \pi W_{0 n}\left(t-\frac{\ell}{W_{n}}\right)} \frac{\sin \pi W_{n}\left(t-\frac{\ell}{W_{n}}\right)}{\pi W_{n}\left(t-\frac{\ell}{W_{n}}\right)}
$$

with $W_{0 n}$ representing the mid band frequency of the $(n, m)^{t h}$ mode. We obtain (12) by substituting $\Psi_{n m}(r, \omega)$ from (11) in (10) and then exchanging integration and summation.

Therefore, following the same reasoning as Shannon [10], the minimum numbers of terms required in the sum (12) that satisfy the constraints on observation region size, bandwidth and observation time window provide the available degrees of freedom of the $(n, m)^{t h}$ mode space-time signal $\psi_{n m}(R, t)$. Note that $\psi_{n m}(R, t)$ can be determined by taking samples
$1 / W_{n}$ units apart and is limited within the interval $T_{\text {eff }}$. This means that we can truncate $\ell$ in (12) to $\left[0, W_{n} T_{\text {eff }}\right]$ and the degrees of freedom of the $(n, m)^{t h}$ mode space-time signal $\psi_{n m}(R, t)$ on the sphere is

$$
D_{n}=W_{n} T_{\text {eff }}+1
$$

where for different values of $n, W_{n}$ is provided in (9) and $T_{\text {eff }}$ is defined in (4).

However, in multi-antenna systems, the information capacity grows linearly with the number of signal degrees of freedom. Therefore, in the following sections, we use the findings of this section to evaluate the information capacity of wideband multipath fields.

\section{Geometrical Representation of The Signals}

From (2), we observe that the gain of the $(n, m)^{t h}$ spatial channel $j_{n}(\omega R / c)$ varies with angular frequency $\omega$. Lets consider a small bandwidth $\Delta W_{n \nu}$ where the gain can be assumed to be constant at $j_{n}\left(\omega_{\nu} R / c\right)$ with $\omega_{\nu}$ representing the mid band angular frequency for the bandwidth $\Delta W_{n \nu}$.

Now, if we consider that the $(n, m)^{t h}$ mode space-time signal $\psi_{n m}(R, t)$ is operating at angular frequency $\omega_{\nu}$ with a bandwidth $\Delta W_{n \nu}$, then (12) can be rewritten as

$$
\psi_{n m}^{(\nu)}(R, t)=\sum_{\ell=-\infty}^{\infty} \psi_{n m}^{(\nu)}\left(R, \frac{\ell}{\Delta W_{n \nu}}\right) \phi_{n \ell}^{(\nu)}(t)
$$

where

$$
\phi_{n \ell}^{(\nu)}(t)=e^{j 2 \pi W_{0 n \nu}\left(t-\frac{\ell}{\Delta W_{n \nu}}\right)} \frac{\sin \pi \Delta W_{n \nu}\left(t-\frac{\ell}{\Delta W_{n \nu}}\right)}{\pi \Delta W_{n \nu}\left(t-\frac{\ell}{\Delta W_{n \nu}}\right)}
$$

with $W_{0 n \nu}$ representing the mid band frequency.

Therefore, we can truncate $\ell$ in (15) to $\left[0, \Delta W_{n \nu} T_{e f f}\right]$ and at frequency $\omega_{\nu}$, the available degrees of freedom of the $(n, m)^{t h}$ mode space-time signal $\psi_{n m}^{(\nu)}(R, t)$ on the sphere is

$$
D_{n \nu}=\Delta W_{n \nu} T_{e f f}+1
$$

Consider a space of $D_{n \nu}$ dimensions, then the $D_{n \nu}$ samples of the signal can always be considered as the coordinates of a point in this space. In this section, we use this geometric idea to map the observable signals in the $D_{n \nu}$ dimensional space.

Note that in (15), at a particular frequency $\omega_{\nu}, \psi_{n m}^{(\nu)}(R, t)$ is represented as a sum of orthogonal functions, since the functions $\phi_{n \ell}^{(\nu)}(t)(16)$ are orthogonal over time [12, eqn. 48]. Thus, by truncating $\ell$ to $\left[0, \Delta W_{n \nu} T_{e f f}\right]$ in (15) and integrating over time, we have

$$
\int_{-\infty}^{\infty}\left|\psi_{n m}^{(\nu)}(R, t)\right|^{2} d t=\frac{1}{\Delta W_{n \nu}} \sum_{\ell=0}^{\Delta W_{n \nu} T_{e f f}} \psi_{n m}^{(\nu)^{2}}\left(R, \frac{\ell}{\Delta W_{n \nu}}\right)
$$

where on the right side,

$$
\left(d_{n m}^{(\nu)}\right)^{2}=\sum_{\ell=0}^{\Delta W_{n \nu} T_{e f f}} \psi_{n m}^{(\nu)^{2}}\left(R, \frac{\ell}{\Delta W_{n \nu}}\right)
$$

is the square of the distance from the origin analogous to two or three dimensional cases, for instance, if $a_{0}, a_{1}$ represents the 
coordinates of a point in the two dimensional space, the square of the distance from the origin is $d_{2}^{2}=a_{0}^{2}+a_{1}^{2}$. Further, on the left side, we have energy $E_{n m}\left(\omega_{\nu}\right)$ of the corresponding signal at radius $R$ operating at a particular frequency $\omega_{\nu}$. Thus, the square of the distance from the origin to a point is

$$
\begin{aligned}
\left(d_{n m}^{(\nu)}\right)^{2} & =\Delta W_{n \nu} E_{n m}\left(\omega_{\nu}\right) \\
& =\Delta W_{n \nu} T_{e f f} P_{n m}\left(\omega_{\nu}\right)
\end{aligned}
$$

where at frequency $\omega_{\nu}, P_{n m}\left(\omega_{\nu}\right)$ is the average power of the $(n, m)^{t h}$ mode space-frequency spectrum at radius $R$ over time $T_{\text {eff }}$ such that

$$
\begin{aligned}
P_{n m}\left(\omega_{\nu}\right) & =E\left\{\left|\alpha_{n m}\left(\omega_{\nu}\right)\right|^{2}\right\}\left|j_{n}\left(\frac{\omega_{\nu}}{c} R\right)\right|^{2} \\
& \leq P\left|j_{n}\left(\frac{\omega_{\nu}}{c} R\right)\right|^{2} .
\end{aligned}
$$

This definition is derived using (2) and afterwards, bounding $E\left\{\left|\alpha_{n m}\left(\omega_{\nu}\right)\right|^{2}\right\}$ by (3).

Hence, at a particular frequency $\omega_{\nu}$, the $(n, m)^{t h}$ mode space-time signal with an average power less than $P_{n m}\left(\omega_{\nu}\right)$ corresponds to a point within a sphere of radius

$$
r_{t}^{(\nu)}=\sqrt{\Delta W_{n \nu} T_{e f f} P_{n m}\left(\omega_{\nu}\right)} .
$$

Lets denote this sphere as Shannon's sphere.

However, in practical systems, signals are perturbed by noise. Hence, the space-frequency spectrum actually observed on the sphere is

$$
\Psi\left(R \hat{\boldsymbol{x}}, \omega_{\nu}\right)=\Psi\left(R \hat{\boldsymbol{x}}, \omega_{\nu}\right)+\eta_{R}\left(\hat{\boldsymbol{x}}, \omega_{\nu}\right)
$$

where $\eta_{R}\left(\hat{\boldsymbol{x}}, \omega_{\nu}\right)$ is the white Gaussian noise on the surface of the spherical region associated with the receive antenna at the angular position $\hat{\boldsymbol{x}}$ and $\Psi\left(R \hat{\boldsymbol{x}}, \omega_{\nu}\right)$ is defined in (1).

Moreover, according to [11, Theorem 2], given a zero mean white Gaussian noise represented by $\eta_{R}\left(\hat{\boldsymbol{x}}, \omega_{\nu}\right)$ and $\hat{\boldsymbol{x}} \in \mathbb{S}^{2}$ lies on the surface of a sphere $\mathbb{S}^{2}$ with variance $\sigma_{0}^{2}\left(\omega_{\nu}\right)$ in $L^{2}\left(\mathbb{S}^{2}\right)$, then for the orthonormal basis functions $Y_{n m}(\hat{\boldsymbol{x}})$, the spatial Fourier coefficients of the noise

$$
\mu_{n m}\left(\omega_{\nu}\right)=\int_{\mathbb{S}^{2}} \eta_{R}\left(\hat{\boldsymbol{x}}, \omega_{\nu}\right) Y_{n m}^{*}(\hat{\boldsymbol{x}}) d \hat{\boldsymbol{x}}
$$

is also a zero mean Gaussian random variable with variance $E\left\{\left|\mu_{n m}\left(\omega_{\nu}\right)\right|^{2}\right\}=\sigma_{0}^{2}\left(\omega_{\nu}\right)$. Since the noise is white Gaussian, the power spectral density of noise is the same at all frequencies $\omega_{\nu}$, i.e.,

$$
E\left\{\left|\mu_{n m}\left(\omega_{\nu}\right)\right|^{2}\right\}=\sigma_{0}^{2} .
$$

and independent of the mode.

Now, we can rewrite (23) as

$$
\Psi\left(R \hat{\boldsymbol{x}}, \omega_{\nu}\right)=\sum_{n=0 m=-n}^{\infty} \sum_{m}^{n}\left[\alpha_{n m}\left(\omega_{\nu}\right) j_{n}\left(\frac{\omega_{\nu}}{c} R\right)+\mu_{n m}\left(\omega_{\nu}\right)\right] Y_{n m}(\hat{\boldsymbol{x}}) .
$$

This expression is derived by substituting (1) and (2) in (23) and then applying (24).

Further, with the presence of white Gaussian noise, we can define the $(n, m)^{t h}$ mode received spectrum at a particular frequency $\omega_{\nu}$ on the surface of the region as

$$
\Psi_{n m}^{(\nu)}\left(R, \omega_{\nu}\right)=\alpha_{n m}\left(\omega_{\nu}\right) j_{n}\left(\frac{\omega_{\nu}}{c} R\right)+\mu_{n m}\left(\omega_{\nu}\right) .
$$

Note that in the $D_{n \nu}$ dimensional space, perturbation of signal by noise during transmission will introduce a small region of uncertainty about each point that represents the $(n, m)^{t h}$ mode signal. As a result, each point that represents the $(n, m)^{t h}$ mode signal will move a certain distance in the space by $\sqrt{\Delta W_{n \nu} T_{\text {eff }}}$ times the rms value of noise. Hence, in the $D_{n \nu}$ dimensional space, the $(n, m)^{t h}$ mode received signal will correspond to a point within a Shannon's sphere of radius

$$
r_{r}^{(\nu)}=\sqrt{\Delta W_{n \nu} T_{e f f}\left(P_{n m}\left(\omega_{\nu}\right)+\sigma_{0}^{2}\right)} .
$$

Moreover, it is evident that for a significantly large $T$, the received signals with an average power $P_{n m}\left(\omega_{\nu}\right)+\sigma_{0}^{2}$ lie on the surface of a Shannon's sphere of radius $r_{r}^{(\nu)}$. Hence, the number of signals distinguishable reliably will certainly not be more than the volume of the Shannon's sphere of radius $r_{r}^{(\nu)}$ divided by the volume of the Shannon's sphere of radius $\sqrt{\Delta W_{n \nu} T_{e f f} \sigma_{0}^{2}}$, since the overlap of the noise sphere results in the uncertainty to the received information. Consider that it is possible to distinguish reliably $M$ different signal functions of duration $T_{\text {eff }}$ (4) on the $(n, m)^{t h}$ channel. Therefore, an upper limit for the number $M$ is

$$
M \leq\left(\sqrt{\frac{P_{n m}\left(\omega_{\nu}\right)+\sigma_{0}^{2}}{\sigma_{0}^{2}}}\right)^{D_{n \nu}},
$$

since the volume of an $q$-dimensional sphere of radius $r$ is given by [10, eqn. 20]

$$
V=\frac{\pi^{q / 2}}{\Gamma(q / 2+1)} r^{q} .
$$

\section{Channel CAPacity}

From a spatial diversity perspective, in Shannon's proposed communication model [10], wideband signals are encoded in only one spatial mode or information is transmitted over one channel. As a result, in our proposed model, there are finite number of independent channels available for information transmission. In this section, we provide a theorem to evaluate the capacity of the $(n, m)^{t h}$ channel.

Before proceeding to do so, note that at a particular frequency $\omega_{\nu}$, capacity of the $(n, m)^{t h}$ channel is

$$
C_{n m}\left(\omega_{\nu}\right)=\lim _{T \rightarrow \infty} \frac{\log _{2} M}{T_{e f f}}
$$

where $M$ and $T_{\text {eff }}$ are defined in (29) and (4), respectively. Hence, we can provide the following theorem to evaluate the capacity of the $(n, m)^{t h}$ channel over the given bandwidth $\left[F_{0}-W, F_{0}+W\right]$.

Theorem 1: Given any signal observed within a multipath wireless field band limited to $\left[F_{0}-W, F_{0}+W\right]$ over the time interval $[0, T]$ on the surface of a spherical region of 
radius $R$, then the capacity of the $(n, m)^{t h}$ channel due to the availability of measurements over the spatial region is

$$
C_{n m} \leq \frac{1}{2} \int_{\Omega_{n}} \log _{2}\left(1+\frac{P\left|j_{n}\left(\frac{\omega}{c} R\right)\right|^{2}}{\sigma_{0}^{2}}\right) d \omega \mathrm{bits} / \mathrm{sec}
$$

with minimal frequency of errors. Here, at a particular frequency $\omega, \sigma_{0}^{2}$ and $P_{n m}(\omega) \leq P\left|j_{n}\left(\frac{\omega}{c} R\right)\right|^{2}$ represent the average noise power and the average signal power of the $(n, m)^{t h}$ channel, respectively. Further, $\Omega_{n} \in\left[2 \pi\left(F_{0}-W\right), 2 \pi\left(F_{0}+W\right)\right]$ if $0 \leq n \leq N_{\min }$ or $\Omega_{n} \in\left[2 \pi F_{n}, 2 \pi\left(F_{0}+W\right)\right]$ if $N_{\min }<n \leq$ $N_{\max }$ where $F_{n}$ is defined in (8).

A detailed proof of the theorem is provided in Appendix A.

\section{A. Discussion}

In this section, we elucidate the following physical insights of the result derived in (32):

- Lets consider that for the $(n, m)^{t h}$ channel, the average noise power and the average signal power over the bandwidth are fixed. Then based on the definition of $W_{n}$ provided in (9), it is evident from (32) that not all the channels have the same capacity. Instead, as $n$ becomes large $C_{n m}$ drops, since the higher modes can utilize only a fraction of the given bandwidth. Thus, the higher modes have less capacity.

- The concept of information transmission in multi-antenna systems is related to the diversity signals carry in both time and space domain. On the contrary, the classical view of Shannon's capacity theorem [10] considers only the time domain along with its transformed counterpart, the frequency spectrum. In this work, we characterize the space-time signals as a sum of orthogonal basis function encoded in finite spatial modes (independent channels) incorporated with Shannon's time domain representation of signals. This characterization portrays the strong coupling between time and space domain for broadband transmission. We use this characterization to evaluate the capacity of each channel (32). Our derived result, thus, indicates how information is conserved in time-space.

- Shannon's work considers broadband transmission over a single channel. However, when spatial diversity is considered, in addition to broadband transmission, there are finite number of channels available. This means that considering spatial diversity, we can capture more information from broadband transmission. Hence, analogous to time, space becomes an information bearing object.

In the following section, we provide more insights of the result derived in (32) based on a numerical example.

\section{B. Graphical Illustration}

We consider transmission for a typical $3 G$ wireless network frequencies (i.e., $F_{0}-W=835 \mathrm{MHz}$ and $F_{0}+W=845 \mathrm{MHz}$ ). Further, the observation region is enclosed within a spherical region of radius $R$ equivalent to twice of the maximum wavelength $\lambda_{\max }$, i.e., $R=2 \lambda_{\max }=2 c /\left(F_{0}-W\right)$ meter with the wave velocity $c=3 \times 10^{8}$ meter per sec. In addition, we keep the ratio $P / \sigma_{0}^{2}$ fixed at $20 \mathrm{~dB}$. Then we plot the capacity of different spatial modes as a function of radius.

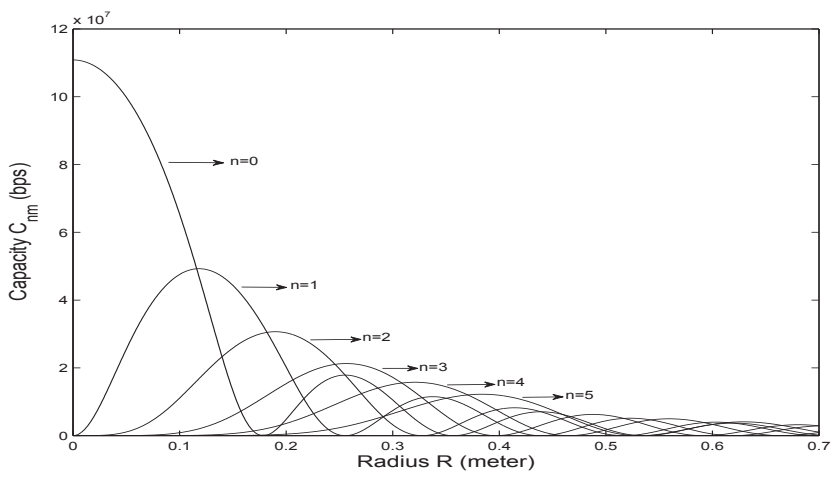

(a)

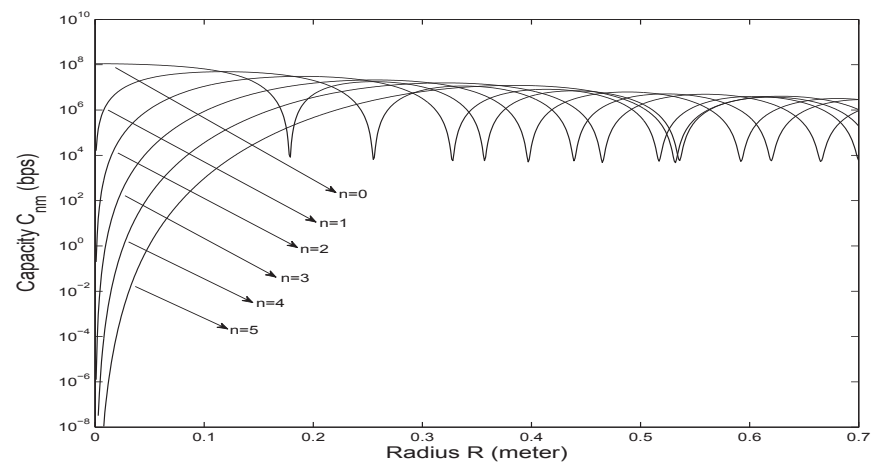

(b)

Fig. 2. Capacity $C_{n m}$ as a function of radius $R$. For each $n,|m| \leq n$. Capacity $C_{n m}$ is in (a) linear scale, (b) log scale.

It is evident from the figure that different modes provide best performance at different radial positions. Moreover, with increasing $n$, capacity starts small due to the asymptotic characteristics of the spherical bessel function $j_{n}(\omega R / c)$ and the optimum performance is obtained at a larger radial position. Further, the maximum capacity achieved at the $(n, m)^{t h}$ mode is greater than the maximum provided by the successor modes.

\section{CONCLUSION}

In this work, we consider a farfield multipath communication system observable on the surface of a spherical region of finite radius. We assume that the wavefiled is band limited and observed over a finite time window. Under these constraints on region size, bandwidth and observation time, the wavefield is expressed as a series of orthogonal basis functions encoded in a finite number of spatial modes. Further, each of these spatial modes represents an independent channel. We show that for a fixed average signal power over the bandwidth, the capacity of all the channels are not same. Our result provides the distribution of the capacity over the different spatial channels. In addition, at different modes optimum capacity is achieved at different radial positions. 


\section{APPENDIX A}

\section{PROOF OF THEOREM 1}

Proof: To prove the capacity expression (32), we must show that the $(n, m)^{t h}$ channel can transmit $1 / 2 \int_{\Omega_{n}} \log _{2}[1+$ $\left.P\left|j_{n}\left(\frac{\omega}{c} R\right)\right|^{2} / \sigma_{0}^{2}\right] d \omega$ bits per sec with a frequency of error less than $\epsilon$ where $\epsilon$ is arbitrarily small. We can prove this with the help of geometry discussed in Section IV.

As discussed in Section IV, at a particular frequency $\omega_{\nu}$,

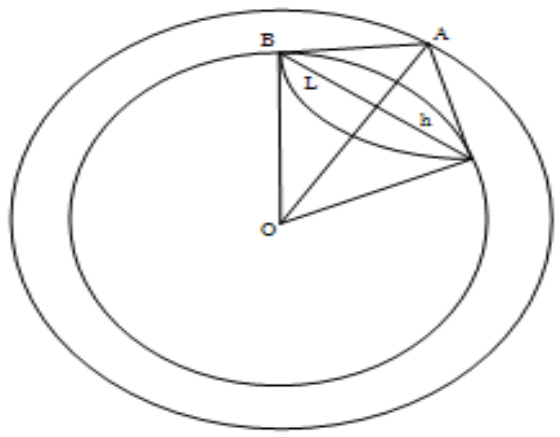

Fig. 3. The geometry involved in deriving (32) where $O A=r_{r}^{(\nu)}, O B=$ $r_{t}^{(\nu)}, h$ in defined in (34) and $O$ is the origin of the region of interest.

the $(n, m)^{t h}$ mode space-time signal $B$ and $(n, m)^{t h}$ mode received signal $A$ lie very close to the surface of highdimensional Shannon's spheres of radius $r_{t}^{(\nu)}$ and $r_{r}^{(\nu)}$, respectively. Hence, the high-dimensional lens-shaped region $L$ is the region of possible signals that might have resulted in $A$, since the distance between $A$ and $B$ is certainly very close to $\sqrt{\Delta W_{n \nu} T_{e f f} \sigma_{0}^{2}}$. However, the volume of $L$ must be smaller than that of a Shannon's sphere of radius $h$. We can determine the value of $h$ by equating the area of the triangle $O A B$ in two different ways as follows

$$
\begin{gathered}
\frac{1}{2} r_{r}^{(\nu)} h=\frac{1}{2} r_{t}^{(\nu)} \sqrt{\Delta W_{n \nu} T_{e f f} \sigma_{0}^{2}} \\
h=\sqrt{\Delta W_{n \nu} T_{e f f} \frac{P_{n m}\left(\omega_{\nu}\right) \sigma_{0}^{2}}{P_{n m}\left(\omega_{\nu}\right)+\sigma_{0}^{2}}} .
\end{gathered}
$$

Hence, any particular signal point (other than the actual cause of $A$ ) may exist within $L$ with a probability less than the ratio of the volume of Shannon's spheres of radius $h$ and $r_{t}^{(\nu)}$. Using (30), the ratio results in

$$
\left(\frac{h}{r_{t}^{(\nu)}}\right)^{D_{n \nu}}=\left(\frac{\sigma_{0}^{2}}{P_{n m}\left(\omega_{\nu}\right)+\sigma_{0}^{2}}\right)^{D_{n \nu} / 2}
$$

Since we consider $M$ signal points on the $(n, m)^{t h}$ channel, if $p$ represents the probability that all expect the actual cause of $A$ are outside $L$, then $p>$ $\left[1-\left(\sigma_{0}^{2} /\left(P_{n m}\left(\omega_{\nu}\right)+\sigma_{0}^{2}\right)\right)^{D_{n \nu} / 2}\right]^{M-1}$.

However, when all these points are outside $L$, the signal is correctly interpreted. Thus, if we make $p$ greater than $1-\epsilon$, the frequency of errors will be less than $\epsilon$. This will be true if

$$
\left[1-\left(\frac{\sigma_{0}^{2}}{P_{n m}\left(\omega_{\nu}\right)+\sigma_{0}^{2}}\right)^{D_{n \nu} / 2}\right]^{M-1}>1-\epsilon .
$$

Now, using the inequality $(1-x)^{n}>1-n x$ for $n>0$ and following some intermediate steps, we rewrite (36) as

$$
(M-1)<\epsilon\left(\frac{P_{n m}\left(\omega_{\nu}\right)+\sigma_{0}^{2}}{\sigma_{0}^{2}}\right)^{D_{n \nu} / 2} .
$$

Then taking $\log$ on both sides, substituting $D_{n \nu}$ by $\Delta W_{n \nu} T_{\text {eff }}+1$ and dividing both sides by $T_{\text {eff }}=T+2 R / c$, we obtain

$$
\begin{aligned}
\frac{\log _{2}(M-1)}{T_{\text {eff }}}< & \frac{\Delta W_{n \nu}}{2} \log _{2}\left(1+\frac{P_{n m}\left(\omega_{\nu}\right)}{\sigma_{0}^{2}}\right) \\
& +\frac{\log _{2}\left(1+\frac{P_{n m}\left(\omega_{\nu}\right)}{\sigma_{0}^{2}}\right)}{2 T_{\text {eff }}}+\frac{\log _{2} \epsilon}{T_{\text {eff }}} .
\end{aligned}
$$

It should be noted that for any fixed value of $\epsilon$, we can specify (38) by taking $T$ sufficiently large, and also have $\log _{2}(M-$ $1) / T_{\text {eff }}$ or $\log _{2} M / T_{\text {eff }}$ as close to $\left(\Delta W_{n \nu} / 2\right) \log _{2}(1+$ $\left.P_{n m}\left(\omega_{\nu}\right) / \sigma_{0}^{2}\right)$ as we desire.

Consequently, capacity of the $(n, m)^{t h}$ channel over the bandwidth $\left[F_{0}-W, F_{0}+W\right]$ is

$$
\begin{aligned}
C_{n m} & \leq \lim _{\nu \rightarrow \infty} \sum_{\nu} \frac{1}{2} \log _{2}\left(1+\frac{P_{n m}\left(\omega_{\nu}\right)}{\sigma_{0}^{2}}\right) \Delta W_{n \nu} \\
& \leq \frac{1}{2} \int_{\Omega_{n}} \log _{2}\left(1+\frac{P_{n m}(\omega)}{\sigma_{0}^{2}}\right) d \omega .
\end{aligned}
$$

Note that when $n>N_{\min }$, for $\Delta W_{n \nu}$ 's that are below the the critical frequency $F_{n}(8)$, the signal is not detectable. As a result, the infinite sum in (39) is replaced by the integration defined only over $\Omega_{n}$ in (40). Afterwards, replacing $P_{n m}(\omega)$ by (21), we can transmit at a rate arbitrarily close to $C_{n m}$ in (32) with an arbitrarily small frequency of errors.

\section{REFERENCES}

[1] I. E. Telatar, "Capacity of multi-antenna gaussian channels," European Transactions on Telecommunications, vol. 10, pp. 585-595, 1999.

[2] C. N. Chuah, D. N. C. Tse, J. M. Kahn, and R. A. Valenzuela, "Capacity scaling in MIMO wireless systems under correlated fading," IEEE Transactions on Information Theory, vol. 48, no. 3, pp. 637-650, March 2002.

[3] T. S. Pollock, T. D. Abhayapala, and R. A. Kennedy, "Introducing space into space-time MIMO capacity calculations," Journal on Telecommunication Systems, vol. 24, pp. 415-436, 2003.

[4] L. W. Hanlen and M. Fu, "Capacity of MIMO channels: A volumetric approach," in IEEE International Conference on Communications (ICC), 2003, pp. 3001-3005.

[5] S. Wei, D. Goeckel, and R. Janaswamy, "On the asymptotic capacity of MIMO systems with antenna arrays of fixed length," IEEE Transactions On Wireless Communications, vol. 4, no. 4, pp. 1608-1621, July 2005.

[6] M. Franceschetti and K. Chakraborty, "Space-time duality in multiple antenna channels," IEEE Transactions on Wireless Communications, vol. 8, no. 4, pp. 1733-1743, April 2009.

[7] M. Franceschetti, M. D. Migliore, and P. Minero, "The capacity of wireless networks: Information-theoretic and physical limits," IEEE Transactions on Information Theory, vol. 55, no. 8, pp. 3413-3424, August 2009. 
[8] E. Bjornson, P. Zetterberg, M. Bengtsson, and B. Ottersten, "Capacity limits and multiplexing gains of MIMO channels with transceiver impairments," IEEE Communications Letters, vol. 17, no. 1, pp. 9194, Jan. 2013.

[9] A. Ozgur, O. Leveque, and D. Tse, "Spatial degrees of freedom of large distributed MIMO systems and wireless ad hoc networks," IEEE Journal on Selected Areas in Communications, vol. 31, no. 2, pp. 202-214, February 2013

[10] C. E. Shannon, "Communication in the presence of noise," Proceedings of the IRE, vol. 37, no. 1, pp. 10-21, Jan. 1949, reprinted in Proceedings of the IEEE, vol. 86, no. 2, pp. 447-457, Feb. 1998.

[11] F. Bashar, S. M. A. Salehin, and T. D. Abhayapala, "Analysis of degrees of freedom of wideband random multipath fields observed over time and space windows," in IEEE Statistical Signal Processing Workshop, Gold Coast, Australia, 29 Jun - 02 Jul 2014, accepted, available at: http://arxiv.org/abs/1405.1153.

[12] — - "Band limited signals observed over finite spatial and temporal windows: An upper bound to signal degrees of freedom," IEEE Transactions On Signal Processing, 2014, submitted, available at: http://arxiv.org.

[13] E. G. Williams, Fourier Acoustics: Sound Radiation and Nearfield Acoustical Holography. New York: Academic Press, 1999.

[14] M. Abramowitz and I. A. Stegun, Eds., Handbook of Mathematical Functions, 10th ed., National Bureau of Standards, US Government Printing Office, Washington, DC, 1972, vol. 55.

[15] T. D. Abhayapala, T. S. Pollock, and R. A. Kennedy, "Characterization of 3d spatial wireless channels," in IEEE 58th Vehicular Technology Conference, VTC 2003-Fall, vol. 1, 2003, pp. 123-127. 